\title{
Rapid progression of mixed neuroendocrine carcinoma-acinar adenocarcinoma of the prostate: A case report
}

\author{
JINGCHAO WEI, XIAOPING ZHENG, LIUXUN LI, WENSU WEI, ZHI LONG and LEYE HE \\ Department of Urology, The Third Xiangya Hospital of Central South University, Changsha, Hunan 410013, P.R. China
}

Received April 1, 2015; Accepted May 13, 2016

DOI: $10.3892 / \mathrm{ol} .2016 .4737$

\begin{abstract}
The current study presents the case of a 78-year-old male with mixed neuroendocrine (NE) carcinoma-acinar adenocarcinoma of the prostate. The patient received endocrine therapy (maximal androgen blockade) after the initial diagnosis resulting in a significant decrease in serum prostate-specific antigen (PSA) level. The patient underwent transurethral resection of the prostate after 6 months of androgen-deprivation therapy for extraordinarily salient difficulty in urination, and histopathology demonstrated mixed NE carcinoma-acinar adenocarcinoma once again. The NE prostate cancer progressed rapidly even though the serum PSA was controlled at a low level on account of the endocrine therapy. Previous treatment protocols were replaced by combined chemotherapy and endocrine therapy, and the patient responded well. The patient's serum PSA remained at a low level, however, urethral dilatation for acute urinary retention was required again 5 months later and the lower urinary tract symptoms became increasingly evident. The patient eventually died of cachexia.. These findings indicate that mixed NE carcinoma-acinar adenocarcinoma has a higher degree of malignancy and that endocrine therapy for prostate cancer has a propensity to facilitate progression of this tumor.
\end{abstract}

\section{Introduction}

Pure primary neuroendocrine (NE) prostate cancer (NEPC) is a rare entity $(<1 \%)$ with an incidence of 35 cases per 10,000 individuals each year (1). The majority of NEPCs originate from conventional prostatic adenocarcinoma, and mixed NE carcinoma-acinar adenocarcinomas of the prostate are

Correspondence to: Dr Leye He, Department of Urology, The Third Xiangya Hospital of Central South University, 138 Tongzipo Road, Changsha, Hunan 410013, P.R. China

E-mail: heleye@aliyun.com

Abbreviations: PSA, prostate-specific antigen; AR, androgen receptor; NE, neuroendocrine

Key words: neuroendocrine prostate cancer, endocrine therapy, rapid progression formed (2). Marcus et al (3) identified 502 cases of NE carcinoma and this study showed that the 5-year overall survival of NE carcinoma was $12.6 \%$ while the 5-year overall survival for non-variant prostate adenocarcinoma was $76.5 \%$. More than one-half of patients presented with metastatic disease at diagnosis and the prognosis of NEPCs was poor. A combination of platinum plus etoposide may be helpful. However, the duration of response was transient (1). Fléchon et al (4) studied carboplatin and etoposide administration in patients with anaplastic progressive metastatic castration-resistant prostate cancer with NE differentiation. The treatment combined carboplatin, with a target area under the plasma concentration versus time curve of 4 , administered over $1 \mathrm{~h}$ on day 1 , and etoposide at a dose of $100 \mathrm{mg} / \mathrm{m}^{2}$ per day administered intravenously over $2 \mathrm{~h}$ for 3 consecutive days. This regimen was repeated every 21 days, with a maximum of 6 cycles. Of 41 patients treated, $13(33 \%)$ obtained a serum NE marker response (>50\% decrease of neuron specific enolase or chromogranin A). This study demonstrates that the association of etoposide and either cisplatin or carboplatin is active in poorly differentiated $\mathrm{NE}$ cancers (4). Another study performed by Fjällskog et al (5) also demonstrates that the combination of cisplatin and etoposide can produce significant responses in patients with heavily pretreated and poorly differentiated/rapidly progressing NE tumors. The present study reports a typical case of mixed NE carcinoma-acinar adenocarcinoma of the prostate. Patients with mixed NE carcinoma-acinar adenocarcinoma of the prostate have rarely been reported. The progression of this tumor is associated with aggressive disease, frequent visceral metastases and a poor prognosis (6). In the current study, the case of a 78-year-old male is presented. Mixed NE carcinoma-acinar adenocarcinoma of the prostate was confirmed by transrectal prostate biopsy due to a 12-month history of urinary frequency and urgency. The patient underwent transurethral resection of the prostate after 6 months of androgen-deprivation therapy for extraordinarily salient difficulty in urination. Invasion of the rectum appeared several months later. The neoplasm progressed extremely fast within a short time. The patient provided written informed consent for publication of this case study.

\section{Case report}

In October 2013, a 78-year-old male presented to the Department of Urology, The Third Xiangya Hospital of Central 


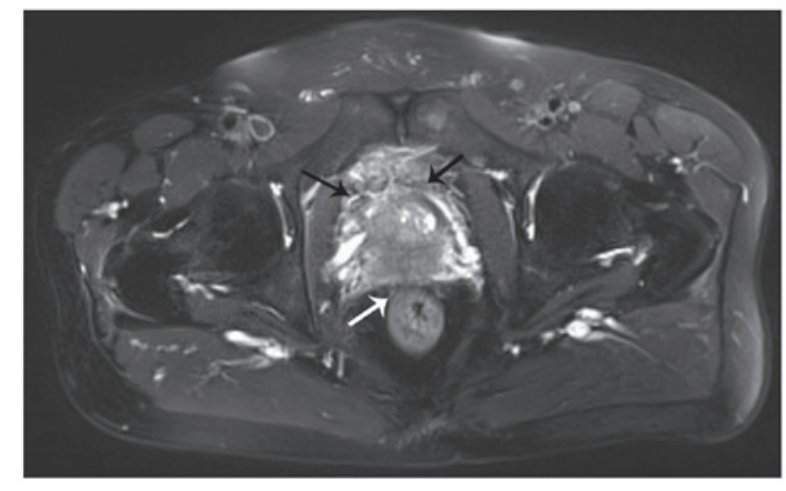

Figure 1. T2-weighted magnetic resonance imaging (transverse section). Nodules of mixed long and short T2 signals were visible around the prostate (black arrow). The rectum was not invaded (white arrow).
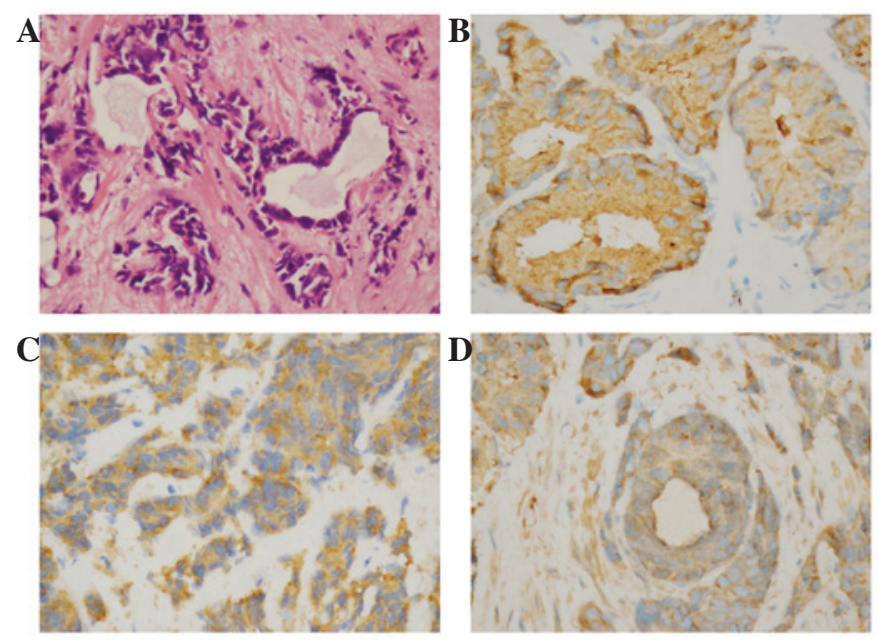

Figure 2. Histology and immunophenotype of the tumor. (A) The major component of the primary tumor consisted of sheets of histologically undifferentiated carcinoma cells with a high nuclear:cytoplasmic ratio. (hematoxylin and eosin staining; original magnification, x200). Immunohistochemical examination of the initial biopsy revealed a poorly-differentiated carcinoma of the prostate that was strongly positive for (B) prostatic acid phosphatase, (C) synaptophysin and (D) chromogranin A (original magnification, x200).

South University (Changsha, China) with a 12-month history of urinary frequency and urgency. The patient denied any difficulty of urination, gross hematuria, bone pain or significant weight loss. Digital rectal examination demonstrated an enlarged, firm prostate, with nodules. The remainder of the physical examination was unremarkable. The patient had no significant medical history other than type II diabetes mellitus. The serum prostate-specific antigen (PSA) level was $33 \mathrm{ng} / \mathrm{ml}$, while the commonly used reference range of PSA is $<4 \mathrm{ng} / \mathrm{ml}$.. Magnetic resonance imaging of the pelvis disclosed the serious possibility of prostate cancer involving the bilateral seminal vesicle glands and urinary bladder, with no lymph node metastasis (Fig. 1). Bone scans via single photon emission computed tomography disclosed metabolic abnormalities on the upper segment of the left femoral bone. A 12-core transrectal ultrasound-guided prostate biopsy was performed.

Histopathology revealed mixed NE carcinoma-acinar adenocarcinoma (Fig. 2). Tissue samples were fixed in $4 \%$ paraformaldehyde, dehydrated in a graded ethanol series (70,

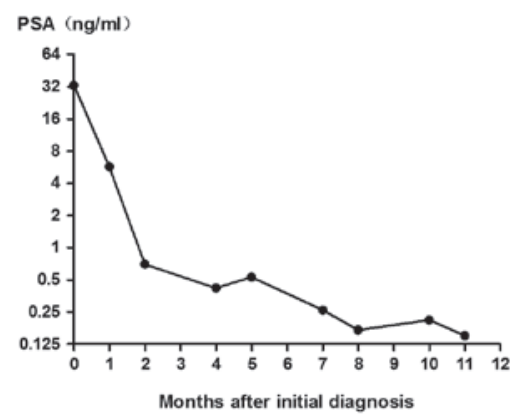

Figure 3. Retrospective review of serum prostate-specific antigen (PSA) levels revealing that the serum PSA levels were not closed correlated with the disease status in the patient. Androgen-deprivation therapy controlled the serum PSA at low levels, whereas the disease progressed rapidly.

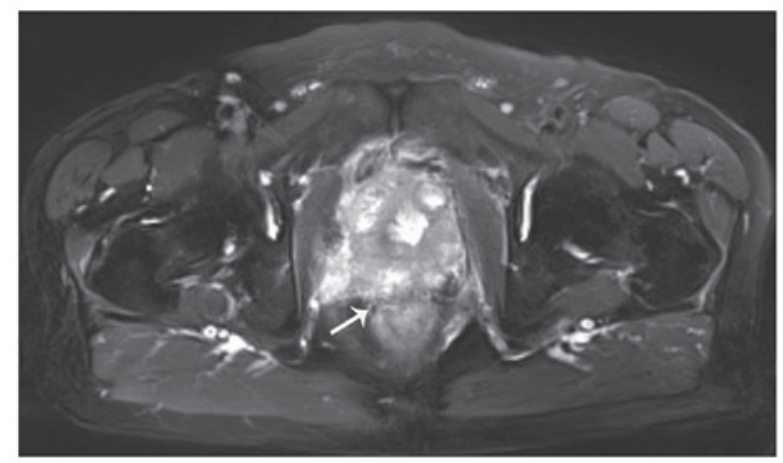

Figure 4. T2-weighted magnetic resonance imaging (transverse section). A markedly swollen prostate was evident, which invaded the rectum (white arrow).

$80,95,95,100$ and 100\%) and paraffin-embedded. Tissue paraffin blocks were sliced into $2.5-\mu \mathrm{m}$ sections after being frozen. Prostate cancer-associated protein markers were detected by hematoxylin-eosin staining and immunohistochemical staining. The antibodies used were as follows: Anti-prostatic acid phosphatase antibody (rabbit monoclonal; dilution, 1:250; catalog no., ab108984; Abcam, Cambridge, UK); anti-synaptophysin antibody (rabbit monoclonal; dilution, 1:400; catalog no., ab32127; Abcam); anti-chromogranin A antibody (rabbit polyclonal; dilution, 1:400; catalog no., ab15160; Abcam); and IgG secondary antibody (goat polyclonal; dilution, 1:100; catalog no. ab175471; Abcam). The hematoxylin-eosin staining revealed poorly differentiated acinar adenocarcinoma in certain areas of the prostate, while the immunohistochemical examination revealed that the tissue sections were positive for prostatic acid phosphatase, synaptophysin and chromogranin A, leading to the diagnosis of mixed NE carcinoma-acinar adenocarcinoma.

The patient was referred to a medical oncologist for therapy of clinical stage T4N0M1b prostate cancer, according to the prostate tumor-node-metastasis staging system of American Joint Committee on Cancer (7th edition) (7). Combined androgen blockade with bicalutamide and triptorelin was initiated (bicalutamide $50 \mathrm{mg}$ daily and triptorelin $15 \mathrm{mg}$ every 3 months, with a total course of 12 months). The patient's PSA level responded well, decreasing to 0.7 and $0.42 \mathrm{ng} / \mathrm{ml}$ after 2 and 5 months of treatment, respectively. Approximately 
1 month later, and 6 months after the initial treatment, the patient presented with difficulty in urination and urinary frequency. The PSA level was rechecked and found to be $0.53 \mathrm{ng} / \mathrm{ml}$ (Fig. 3). Transrectal ultrasonography estimated the volume of the prostate to be larger than previously. A plasmakinetic resection of the prostate was performed. Combined chemotherapy based on etoposide and carboplatin was then initiated simultaneously with the endocrine therapy (etoposide $100 \mathrm{mg}$ daily for 3 consecutive days, repeated every 4 weeks, and carboplatin injection $400 \mathrm{mg}$ once, repeated every 4 weeks, with a total course of 6 months). The serum PSA remained at a low level $(<0.5 \mathrm{ng} / \mathrm{ml})$ from then on. Conversely, the patient's clinical discomfort progressed markedly (Fig. 4).

Approximately 5 months after the combined chemotherapy and endocrine therapy, the patient presented with acute urinary retention and underwent urethral dilatation. Subsequently, 12 months after the initial diagnosis, the patient's condition deteriorated. In addition to serious lower urinary tract symptoms, the patient presented with urinary tract infection and kidney failure, although the serum PSA remained at a low level $(<0.7 \mathrm{ng} / \mathrm{ml})$. Endocrine therapy was terminated and docetaxel (120 mg injection, every 4 weeks for 3 months) was used to replace etoposide and carboplatin. However, those measures had little effect on the patient's serious lower urinary tract symptoms. Finally, 18 months subsequent to the initial diagnosis, the patient succumbed to cachexia.

\section{Discussion}

The majority of conventional prostate cancers are prostatic adenocarcinomas, which are formed from tumor cells with luminal differentiation that express androgen receptors (ARs) and secrete PSA. Therefore, hormonal therapy based on AR-dependent signaling that results from AR gene amplification, constitutive activation of AR, intratumoral androgen production or other mechanisms is used in patients with prostatic adenocarcinoma at the time of initial diagnosis, and achieves a therapeutic effect in nearly all cases (8). Even castration-resistant prostate cancer shows progression via AR-dependent signaling mechanism (9). The patient in the present case experienced rapid progression following the diagnosis of mixed NE carcinoma-acinar adenocarcinoma of the prostate.

NEcells exist in normal prostate tissues, without expressing AR or secreting PSA. Extensive focal NE differentiation is present in $10 \%$ of all conventional adenocarcinomas (10). It has been proposed that androgen-deprivation therapy may facilitate progression of pre-existing prostatic adenocarcinoma to NE prostate cancer. A consequence of a lack of ARs is that deprivation hormonal therapy does not eliminate NE cells (11). This phenotype composed of aggressive and highly proliferative NE cells shows conspicuous clinical progression with a low serum PSA level. Kinebuchi et al (12) reported a case of relapsed prostate cancer with high serum levels of carcinoembryonic antigen and pro-gastrin-releasing peptide, without elevation of serum PSA after bicalutamide and leuprorelin acetate combined hormonal therapy for 39 months. The patient finally succumbed after a survival period of 11 months following the clinical progression. The present study patient underwent transurethral resection of the prostate due to difficulty in urination after receiving endocrine therapy for 6 months, and the pathological examination indicated mixed NE and prostatic adenocarcinoma once again.

The international classification standard for NE prostate cancer is not yet clear. According to the Prostate Cancer Foundation, the pathological classification system for prostatic cancer NE differentiation consists of the following: i) Usual prostate adenocarcinoma with NE differentiation; ii) adenocarcinoma with Paneth cell NE differentiation; iii) carcinoid tumors; iv) small cell carcinoma; v) large cell NE carcinoma; and vi) mixed NE carcinoma-acinar adenocarcinoma (13). In the present case, the patient was diagnosed with mixed NE carcinoma-acinar adenocarcinoma. Tanaka et al (14) reported the following characteristics for the clinical course of NE prostate cancer: i) Survival time is short subsequent to relapse; ii) PSA levels do not increase subsequent to relapse; and iii) the metastatic sites are similar to those of common adenocarcinomas. Marcus et al (15) reported a median survival time of 10 months for patients with NE tumors and a 5-year overall survival rate of $12.6 \%$. Given that NE prostate cancer is independent of AR signaling, combination chemotherapy must be added to the current therapeutic regimen. Yashi et al (16) reported that combination chemotherapy based on cisplatin and etoposide was effective for controlling small cell NE carcinoma occurring after use of a luteinizing hormone-releasing hormone analogue and bicalutamide for 10 months in a 69-year-old patient who had experienced progression for 7 months (16). In the present case, the patient underwent a transurethral resection of the prostate for progressive dysuria after 6 months of androgen-deprivation therapy. The patient then received combined chemotherapy and endocrine therapy 2 months later. After another 5 months, the patient experienced urinary retention and underwent urethral dilatation.

In the present case, endocrine therapy of mixed NE carcinoma-acinar adenocarcinoma of the prostate had a propensity to facilitate progression of the tumor. Since patients who have undergone failed hormonal therapy often present with multiple metastases and a poor prognosis (17), biopsies or resections have rarely been performed in such patients unless the entity has progressed to severe dysuria. Therefore, the assessment of the incidence of androgen-deprivation therapy-induced NE prostate cancer has been neglected, making such cases underdiagnosed (18). In consequence of the widespread use of novel androgen axis-targeting drugs, such as abiraterone acetate and enzalutamide (19), the incidence of NE prostate cancer may increase to a great extent.

In summary, the present study reported a case of rapid progression in a mixed NE carcinoma-acinar adenocarcinoma of the prostate. In patients diagnosed with mixed NE carcinoma-acinar adenocarcinoma of the prostate, endocrine therapy shows a poor effect, even if the serum PSA level has remained low, and a combination chemotherapy may be useful to control the progression. Close follow-up of symptoms and periodic digital rectal examination are also important.

\section{References}

1. Aggarwal R, Zhang T, Small EJ and Armstrong AJ: Neuroendocrine prostate cancer: Subtypes, biology, and clinical outcomes. J Natl Compr Canc Netw 12: 719-726, 2014. 
2. Wang HT, Yao YH, Li BG, Tang Y, Chang JW and Zhang J: Neuroendocrine Prostate Cancer (NEPC) progressing from conventional prostatic adenocarcinoma: Factors associated with time to development of NEPC and survival from NEPC diagnosis - a systematic review and pooled analysis. J Clin Oncol 32: 3383-3390, 2014.

3. Marcus DM, Goodman M, Jani AB, Osunkoya AO and Rossi PJ: A comprehensive review of incidence and survival in patients with rare histological variants of prostate cancer in the United States from 1973 to 2008. Prostate Cancer Prostatic Dis 15: 283-288, 2012.

4. Fléchon A, Pouessel D, Ferlay C, Perol D, Beuzeboc P, Gravis G, Joly F, Oudard S, Deplanque G, Zanetta S, et al: Phase II study of carboplatin and etoposide in patients with anaplastic progressive metastatic castration-resistant prostate cancer (mCRPC) with or without neuroendocrine differentiation: Results of the French Genito-Urinary Tumor Group (GETUG) P01 trial. Ann Oncol 22: 2476-2481, 2011.

5. Fjällskog ML, Granberg DP, Welin SL, Eriksson C, Oberg KE, Janson ET and Eriksson BK: Treatment with cisplatin and etoposide in patients with neuroendocrine tumors. Cancer 92: 1101-1107, 2001

6. Palmgren JS, Karavadia SS and Wakefield MR: Unusual and underappreciated: Small cell carcinoma of the prostate. Semin Oncol 34: 22-29, 2007.

7. Chung MS, Lee SH, Lee DH and Chung BH: Evaluation of the 7th American Joint Committee on cancer TNM staging system for prostate cancer in point of classification of bladder neck invasion. Jpn J Clin Oncol 43: 184-188, 2013.

8. Lipianskaya J, Cohen A, Chen CJ, Hsia E, Squires J, Li Z, Zhang Y, Li W, Chen X, Xu H and Huang J: Androgen-deprivation therapy-induced aggressive prostate cancer with neuroendocrine differentiation. Asian J Androl 16: 541-544, 2014.

9. Beltran H, Tagawa ST, Park K, MacDonald T, Milowsky MI, Mosquera JM, Rubin MA and Nanus DM: Challenges in recognizing treatment-related neuroendocrine prostate cancer. J Clin Oncol 30: e386-e389, 2012.
10. Di Sant'Agnese PA and Cockett AT: The prostatic endocrine-paracrine (neuroendocrine) regulatory system and neuroendocrine differentiation in prostatic carcinoma: A review and future directions in basic research. J Urol 152: 1927-1931, 1994.

11. Ramírez-Balderrama L, López-Briones S, Daza-Benítez L, Macías MH, López-Gaytán T and Pérez-Vázquez V: Neuroendocrine differentiation in prostate adenocarcinoma. Gac Med Mex 149: 639-645, 2013 (In Spanish).

12. Kinebuchi Y, Noguchi W, Irie K, Nakayama T, Kato H and Nishizawa O: Relapsed prostate cancer with neuroendocrine differentiation and high serum levels of carcinoembryonic antigen without elevation of prostrate-specific antigen: A case report. Int J Urol 14: 147-149, 2007.

13. Epstein JI, Amin MB, Beltran H, Lotan TL, Mosquera JM, Reuter VE, Robinson BD, Troncoso P and Rubin MA: Proposed morphologic classification of prostate cancer with neuroendocrine differentiation. Am J Surg Pathol 38: 756-767, 2014.

14. Tanaka M, Suzuki Y, Takaoka K, Suzuki N, Murakami S, Matsuzaki $\mathrm{O}$ and Shimazaki J: Progression of prostate cancer to neuroendocrine cell tumor. Int J Urol 8: 431-437, 2001.

15. Marcus DM, Goodman M, Jani AB, Osunkoya AO and Rossi PJ: A comprehensive review of incidence and survival in patients with rare histological variants of prostate cancer in the United States from 1973 to 2008. Prostate Cancer Prostatic Dis 15: 283-288, 2012.

16. Yashi M, Terauchi F, Nukui A, Ochi M, Yuzawa M, Hara Y and Morita T: Small-cell neuroendocrine carcinoma as a variant form of prostate cancer recurrence: A case report and short literature review. Urol Oncol 24: 313-317, 2006.

17. Reichardt P: Systematic hormone- and chemotherapy in the management of skeletal metastases. Orthopade 27: 240-244, 1998 (In German)

18. Lipianskaya J, Cohen A, Chen CJ, Hsia E, Squires J, Li Z, Zhang Y, Li W, Chen X, Xu H and Huang J: Androgen-deprivation therapy-induced aggressive prostate cancer with neuroendocrine differentiation. Asian J Androl 16: 541-544, 2014.

19. Aragon-Ching JB: The evolution of prostate cancer therapy: Targeting the androgen receptor. Front Oncol 4: 295, 2014. 